\title{
BMJ Open Predictors of placebo response to local (intra-articular) therapy in osteoarthritis: an individual patient data meta- analysis protocol
}

\author{
Shirley Pei-Chun Yu, ${ }^{1,2}$ Manuela L Ferreira, ${ }^{2}$ Marienke van Middelkoop, ${ }^{3}$ \\ Sita M A Bierma-Zeinstra, ${ }^{4}$ Weiya Zhang, ${ }^{5}$ Leticia A Deveza, ${ }^{1,2}$ David J Hunter ${ }^{\oplus, 2}$
}

To cite: Yu SP-C, Ferreira ML, van Middelkoop $\mathrm{M}$, et al. Predictors of placebo response to local (intra-articular) therapy in osteoarthritis: an individual patient data metaanalysis protocol. BMJ Open 2019;9:e027372. doi:10.1136/ bmjopen-2018-027372

- Prepublication history and additional material for this paper are available online. To view these files, please visit the journal online (http://dx.doi. org/10.1136/bmjopen-2018027372).

Received 19 October 2018 Revised 29 March 2019 Accepted 3 April 2019

Check for updates

(C) Author(s) (or their employer(s)) 2019. Re-use permitted under CC BY-NC. No commercial re-use. See rights and permissions. Published by BMJ.

For numbered affiliations see end of article.

Correspondence to Dr Shirley Pei-Chun Yu; shirleyyu@uni.sydney.edu.au

\section{ABSTRACT}

Introduction 0steoarthritis $(O A)$ is a highly prevalent and disabling condition with limited safe and effective treatment options. Intra-articular therapies are increasingly being used, however whether the effect of these agents is due to active treatment or placebo remains unclear. As the placebo response can be attributed to multiple factors, assessment of the placebo response using individual patient data (IPD) meta-analysis will give insight into the different modifiers of response to placebo. The aim of this IPD meta-analysis is to investigate the predictors of placebo response in intra-articular injection trials in OA. IPD meta-analysis is considered to be superior to conventional meta-analysis, as it combines multiple trial data, facilitates the standardisation of analyses across different studies and allows measuring derivation of the desired information.

Method and analysis A systematic literature search will be conducted for randomised clinical trials comparing corticosteroid and viscosupplementation/hyaluronic acid intra-articular injections with placebo for knee and hip OA. Pubmed (Medline), EMBASE, Web of Science, Cochrane Central and SCOPUS will be searched from inception to September 2018. Corresponding authors of the original trials will be contacted to obtain IPD. Risk of bias will be assessed using the Cochrane Collaboration's tool. The primary outcome will be change in pain from baseline. Secondary outcomes will be change in function and patient's global assessment. Potential predictors of placebo response assessed will include patient's characteristics, pain mechanism characteristics, radiographic severity, pain severity, intervention characteristics and trial design characteristics. A multilevel logistic regression analyses will be applied. Results will be reported using the Preferred Reporting Items for Systematic review and Meta-Analysis -IPD guidelines.

Ethics and dissemination This study does not include identifiable data and ethical approval was obtained by the original investigators. Results of the IPD meta-analysis will be disseminated for publication in peer-reviewed journals and conference presentations.

PROSPERO registration number CRD42018095188

\section{Strengths and limitations of this study}

- The use of an individual patient data meta-analysis of randomised controlled trials (RCTs) will provide more precise estimates of the placebo response. It also allows the identification of patient-level predictors of placebo response in this population.

- The study will be conducted within the framework of the $O A$ Trial Bank, an international organisation that initiates meta-analyses of effect on predefined subgroups of $O A$ patients from existing trials.

- Identification of the predictors of placebo response in intra-articular injections for OA may influence future clinical trial designs with a more tailored approach when classifying participants in future studies.

- Inclusion of frequently utilised intra-articular injection RCTs will allow for a larger sample size, increased precision of the results and provide insight into the more commonly used injectables.

- We have only included injections of corticosteroid and viscosupplements/hyaluronic acid trials because these are the standard intra-articular treatments for $\mathrm{OA}$. There are other intra-articular injection treatments such as blood products, growth factors and prolotherapy. As they are not established treatments with limited evidence in $\mathrm{OA}$, we will exclude them from this study.

\section{INTRODUCTION}

Osteoarthritis (OA) is a highly prevalent condition that imposes a substantial burden on the individuals affected. It is estimated that by $2030,25 \%$ of the population of the USA (67million adults) will have OA. ${ }^{1}$ Current management strategies suggest a focus towards conservative therapies including physiotherapy and weight loss, as well as pain palliation whether it is in the form of medications or ultimately joint replacement surgery. ${ }^{2}$ However, for patients, especially with only symptomatic monoarthritis or oligoarthritis, the systematic effects of oral medications raises safety concerns. ${ }^{3-6}$ 
Intra-articular injection therapies appear to be an attractive alternative in these patients, and there is a trend in the development of investigational intra-articular agents, aiming to improve symptoms and potentially alter disease progression.

Presently available intra-articular therapies are corticosteroids and viscosupplements (hyaluronic acid). ${ }^{78}$ Agents such as blood-derived products are also available in some countries. However, based on current guidelines for knee OA, intra-articular injections are not first-line therapies and are preferred as the last non-operative alternative where other conservative modalities have failed, or in some published treatment guidelines, not recommended at all based on their limited evidence, or controversial efficacy profiles. ${ }^{9} 10$

There are a number of methodological limitations of clinical trials in OA that have constrained progress. Especially with intra-articular therapies in $\mathrm{OA}$, most trials are small, thus affecting the strength of the studies. Another issue is the frequent practice of comparing one controversial agent versus another (ie, platelet-rich plasma versus hyaluronate agent), which will not justify the agent to be superior in the overall treatment of OA. Furthermore, in intra-articular therapy trials, there are concerns of whether intra-articular injection of normal saline should be considered as the ideal agent to be employed as a placebo. There are increasing number of studies contributing to the evidence of intra-articular saline having a potential biological effect. The biological effect in this setting is likely secondary to neurobiological mechanisms such as those via endogenous opioid and dopaine, ${ }^{11}$ as well as via possible dilution of the inflammatory element in the joint because of the volume of saline. ${ }^{12} 13$ Thus, intra-articular saline as a placebo can be considered to be an 'impure placebo' in the context of a placebo-controlled intra-articular injection trial. The inclusion of a no-treatment/sham-injection group may be a way to discern the placebo effect of saline injections, however the presence of this design is rare in OA clinical trials. Despite this, previous meta-analysis of randomised controlled trials (RCTs) assessing the placebo response across a range of therapies in OA (non-pharmacological, pharmacological and surgical treatments) have confirmed that placebo response (effect size (ES) $=0.5195 \%$ CI 0.46 to 0.55 ) is greater than no treatment or spontaneous response (ES=0.03, 95\% CI -0.13 to 0.18 ) for pain in OA. ${ }^{14}$

The inability to demonstrate a minimum clinically important difference (MCID) over placebo, directly affects the development of potential pharmacological innovations and their translation to becoming commercially available treatment options for this disabling disease. The magnitude of the placebo response in OA trials is significant with about $75 \%$ of treatment effect being attributable to placebo contextual effects. ${ }^{15}$ In general, the more invasive and more frequent the administration of an intervention, the larger the placebo response. For invasive therapies, patients' expectations and beliefs create even larger placebo/contextual effects. ${ }^{14}$ When considering clinical trial design, the challenges of which placebo to choose, its volume, injection frequency, the use of injection guidance, concomitant local anaesthetic use, patient baseline disease presentation (bilateral vs unilateral disease, concomitant presence of inflammatory features/effusion, disease severity, baseline pain) all create substantial opportunity for heterogeneity in what is already a challenging clinical trial environment. The intervention itself is also subject to contextual effects; administration route, colour, branding and cost all have an effect, thus indicating that clinical trials may need more standardisation across the board to optimise the demonstration of treatment response. ${ }^{16}$

To date, placebo responses from clinical trials are ultimately measured as a change in outcome from baseline in the placebo group in comparison to the treatment group and is potentially confounded by spontaneous effects such as the Hawthorne effect (ie, the effect due to being observed), natural fluctuation of disease and regression to the mean. ${ }^{14} 16$ Minimal trials incorporate a no-treatment group, which may allow for adequate clarification of the placebo effect. Meta-analysis of OA treatments has shown that the placebo response varies greatly between individuals. ${ }^{15}$ The main limitation of aggregate data meta-analysis is that the variations of the treatment/placebo responses across individuals cannot be scrutinised. As the placebo response can be attributed to the individual or related to the study protocol, assessment of the placebo response utilising individual patient data (IPD) meta-analysis will give insight into the different predictors of placebo response. IPD analysis is now increasingly used over established meta-analysis and is considered to be superior, as it facilitates standardisation of analyses across different studies and allow derivation of the desired information. ${ }^{17}$ Our IPD meta-analysis will examine the role of potential placebo response modifiers, assessing patient, intervention and trial characteristics-contextual factors that are rarely measured and reported in clinical trials or analysed in existing meta-analyses.

This analysis will be conducted under the auspices of the OA Trial Bank, an international collaboration that is endorsed by the Osteoarthritis Research Society International and the European League Against Rheumatism (EULAR). The OA Trial Bank was initiated in 2010 with the purpose of collecting and analysing IPD of published RCTs in OA to identify specific responseive subgroups for the different OA treatment. It brings together data from individuals with a diagnosis of $\mathrm{OA}$, recruited for published RCTs from around the world to form a databank. ${ }^{18} 19$

Therefore, the aim of this IPD analysis is to investigate the predictors of placebo response in intra-articular injection trials in OA. This study will differ from the recently submitted IPD-meta-analysis protocol assessing placebo response in OA by University of Nottingham arthritis research group. ${ }^{19}$ Based on their published protocol, their data extraction from the OA Trial Bank is targeted at OA therapies namely topical non-steroidal anti-inflammatory drugs, topical capsaicin, glucosamine and intra-articular 
glucocorticoids. Potential placebo response modifiers that will be assessed are: patient baseline characteristics (age, gender and body mass index), disease (radiographic information, signs of inflammation, muscle strength, duration of complaints, pain severity, type of pain, central sensitisation and psychological assessments), placebo (oral, topical, injection and dose) and trial and outcome measures (pain, function, patient global assessment and quality of life).${ }^{19}$ In contrast, intra-articular injection therapies will be the only therapies of interest in this analysis. While there will be some cross over regarding patient-level characteristics, the incorporation of viscosupplementation/hyaluronic acid trials and an updated systematic review with the acquisition of newer glucocorticoid trials which will allow for a larger sample size, increased precision of the results and provide insight into the more commonly used injectables. In addition to patient-level characteristics, there will be a focus on interventional and trial characteristics, that is, intervention characteristics (aspirate volume, frequency of injection, volume of injection and intra-articular injection approach) and trial characteristics (clinical setting, blinding, use of intention to treat analysis and funder/sponsor).

\section{METHODS AND ANALYSIS}

IPD from trials comparing intra-articular injection to placebo for knee OA will be extracted and reanalysed to ascertain the magnitude of the placebo response and the role of potential predictors in these trials. The analysis will be conducted under the umbrella of the OA Trial Bank.

The IPD meta-analysis will be conducted in accordance with the methods recommended by the IPD Meta-analysis Methods Group. ${ }^{17}$ Reporting of the meta-analysis will conform with the Preferred Reporting Items for Systematic review and Meta-Analysis (PRISMA)-IPD checklist. ${ }^{20}$

The research question and study proposal of this study has been approved by the steering committee of the OA Trial Bank, before the development of the full study protocol.

\section{Participants}

Participants from the identified RCTs must have a diagnosis of knee and hip OA, according to the criteria defined by the American College of Rheumatology, EULAR evidence-based recommendations for the diagnosis of knee $\mathrm{OA}^{21} 22$ or fulfil specified radiological criteria of OA diagnosis.

\section{Types of baseline assessments}

Participant baseline characteristics including age, gender, bilateral versus unilateral disease, other joint OA involvement, radiographic severity, pain severity at baseline and presence of inflammatory features (based on imaging and physical examination). Intervention characteristics (clinical setting, aspirate volume, frequency of injection, volume of injection and intra-articular injection approach) and trial design characteristics (blinding, dropout rate, use of intention to treat analysis, role of funder/sponsor) will also be extracted.

\section{Types of outcomes}

The primary outcome of the IPD meta-analysis will be change in pain over time. Visual analogue scale (VAS) pain score will be preferentially used for the analysis. If unavailable, the Western Ontario and McMaster Universities Osteoarthritis Index (WOMAC) pain score will be used and converted into a VAS 0-100 scale as per previous OA Trial Bank Protocols. ${ }^{23}$

Secondary outcomes will be a change in function and patient global assessment.

\section{Language}

No language restrictions will apply.

\section{Literature search}

Identification of studies

A systematic literature search will be conducted using the following databases: Pubmed (Medline), EMBASE, Web of Science, Cochrane Central and SCOPUS. The search will be from inception to September 2018. The search strategy was developed by the reviewers in consultation with the OA Trial Bank (online supplementary appendix 1).

Literature searches will be done separately for intra-articular glucocorticoid and viscosupplementation/hyaluronic acid. The literature search approach will comprise of an amalgamation of main search terms including identification of the OA population group, intervention of intra-articular glucocorticoid and viscosupplementation/ hyaluronic acid and of RCT design. Furthermore, efforts will be made to identify unpublished trials through Clinicaltrials.gov, European Union Clinical Trials Register and International Standard Randomised Controlled Trials Number (ISRCTN) registry and contacting pharmaceutical suppliers.

Identified studies will be imported to EndNote X8 for screening.

\section{Screening process}

Studies eligible for inclusion will be assessed by two independent reviewers (SY and LD). Titles and abstracts for potential studies will be screened first, and subsequently, the full text of the selected studies will be reviewed for appropriateness to be included. If no consensus is reached, a third reviewer will be consulted (DJH). The results will be summarised as per the PRISMA guidelines. ${ }^{24}$

\section{Type of studies}

Randomised placebo-controlled trials of intra-articular glucocorticoids and/or viscosupplementation/hyaluronic acid in knee or hip OA will be included. Studies related to inflammatory arthritis (such as rheumatoid or psoriatic arthritis) will be excluded. Animal model and biomarker studies will be excluded. Trials that are not randomised, literature or systematic reviews, and 
conference abstracts without available data will be excluded.

\section{Data collection and transfer}

As per all other studies conducted by the OA Trial Bank, ${ }^{18} 2325$ the same method for data acquisition and transfer will be utilised. The corresponding authors of eligible trials will be invited to collaborate. Initial contact will be by email with two further successive email reminders. If the corresponding author is uncontactable, communication will be attempted with the other trial authors and/or institutions listed. Authors who are willing to collaborate will be asked to sign a data delivery agreement from the OA Trial Bank. This will include items of input data, ownership of data, obligation, terms, authorship and subsequent publication intentions. The data obtained will be stored on a secure server at Erasmus MC University Medical Center, Rotterdam, the Netherlands, and participant details will be kept in an anonymous and confidential fashion. Data quality will be ensured through independent checking looking at data-entry mistakes and inconsistencies. Data received will be compared with the published summary results from the primary studies. In situations where there are differences found, the authors will be contacted to resolve the discrepancy issue.

With the existing intra-articular glucocorticoid trials that have been stored in the OA Trial Bank, the corresponding authors will be contacted and will be asked to sign a further data transfer agreement for the use of their data for the purpose of this analysis.

\section{Patient and public involvement}

There have been no patient and/or public involvement in the design of this IPD meta-analysis.

\section{Risk and quality assessment}

The included trials will be assessed independently by two reviewers to assess the quality of evidence and the risk of biases through the use of the Cochrane Collaboration's tool. ${ }^{26} 27 \mathrm{~A}$ third reviewer will be consulted if there is a disagreement. The domains assessed will include randomisation of procedure, blinding of participants, physicians and treatment allocation, use of intention to treat analysis, incomplete outcome data, baseline group similarity, reporting bias and other sources of biases. Studies will be categorised as 'low risk', 'high risk' or 'unclear. As per previous studies with the OA trial bank, a low risk of bias study will be classified as fulfilling at least 6 of the 12 items in the Cochrane Collaboration's tool. ${ }^{27}$

\section{Data analysis}

A descriptive evaluation of each trial and study participants will be conducted. Publication bias will be investigated using a funnel plot analysis as this will specify the potential impact of both known and unknown missing trials on the results. ${ }^{27}{ }^{28}$ Missing data will be assumed to be missing at random, thus patient characteristics will be used to impute missing data by means of multiple imputation at random. ${ }^{29} 30$ In addition, we will compare the ESs pooled from those responded versus the overall (ie, the ES pooled from all trials systematically searched from the literature) to examine the deviation.

Baseline and follow-up data from the placebo arm will be used to estimate the predictors of the placebo response. Separate analyses will be conducted for glucocorticoids and viscosupplementation/hyaluronic acid, as well as different outcome measures (ie, pain, function and patient global assessment). Trials will also be grouped by type of joint (ie, knee or hip) and follow-up duration (eg, $<4$ weeks or $\geq 4$ weeks for corticosteroid and $<12$ weeks or $\geq 12$ weeks for viscosupplementation/hyaluronic acid).

A one-step approach will be applied, via the use of multilevel regression models to assess for predictors of the placebo response. The use of the one step approach in this setting will allow for a more cohesive modelling of

Table 1 Potential placebo response modifiers

\begin{tabular}{|c|c|}
\hline Study features & Description \\
\hline \multirow[t]{5}{*}{ Patient domain } & Age \\
\hline & Gender \\
\hline & Body mass index \\
\hline & Bilateral versus unilateral disease \\
\hline & Disease duration \\
\hline \multirow{8}{*}{$\begin{array}{l}\text { Pain } \\
\text { mechanisms }\end{array}$} & Central pain mechanisms: \\
\hline & Osteoarthritis in other joints. \\
\hline & Comorbidities. \\
\hline & Pain severity. \\
\hline & Peripheral pain mechanisms: \\
\hline & Radiographic information. \\
\hline & $\begin{array}{l}\text { Presence of inflammatory features } \\
\text { (ultrasound versus physician assessed } \\
\text { joint swelling). }\end{array}$ \\
\hline & Morning stiffness symptoms. \\
\hline \multirow{14}{*}{$\begin{array}{l}\text { Intervention } \\
\text { characteristics }\end{array}$} & Clinical setting (ie, location of intervention) \\
\hline & Aspirate volume \\
\hline & Frequency of injection \\
\hline & Volume of injection \\
\hline & $\begin{array}{l}\text { Intra-articular injection approach (ie, medial } \\
\text { vs lateral approach, use of ultrasound } \\
\text { guided injection) }\end{array}$ \\
\hline & Blinding \\
\hline & Dropout rates per group \\
\hline & Inclusion of a 'no treatment' group \\
\hline & Use of 'intention to treat analysis' \\
\hline & Randomisation ratio \\
\hline & Trial duration \\
\hline & Single centre/multicentre study \\
\hline & Parallel/crossover trial \\
\hline & $\begin{array}{l}\text { Funding/sponsor (ie, pharmaceutical } \\
\text { funding) }\end{array}$ \\
\hline
\end{tabular}


covariates and account for the clustering of participants within the study. ${ }^{17}$ This will be done by combining all the data from all the studies available after appropriate standardisation of the variables and a new dataset will be formed to allow for further analysis. To assess for the potential subgroup effects, a random effect model will be utilised given the hierarchical nature of the data to assess the interaction effects, with change in pain being a dependent variable and potential predictors being independent variables. In the setting where a no-treatment control is available, we will include placebo-no-treatment as an independent variable. Responders to placebo will be compared with non-responders to identify predictors of response.

The primary outcome will be change in pain from baseline and will be determined as the dependent variable in the regression model. The MCID threshold will be a $20 \%$ or more reduction in pain based on the VAS pain score with $0 \mathrm{~mm}$ being no pain to $100 \mathrm{~mm}$ being the worst pain ever. This level has been recommended for use in pain and function assessment in rheumatic diseases such as $\mathrm{OA},{ }^{3132}$ and we will use it to define the placebo response which is equivalent to an ES of $0.8,{ }^{33}$ that indicates the response unlikely to be caused by spontaneous effects. In situations where WOMAC pain score is only available, it will be used instead.

Secondary outcomes will be a change in function and patient global assessment. Change in pain will be determined as the dependent variable, and independent variables will be the potential predictors of placebo response. These will be grouped as patient-level characteristics, peripheral pain mechanisms, central pain mechanisms, intervention characteristics and those related to trial design (blinding, funder/sponsor roles and intention to treat) (table 1) and are as listed below. Each group will be forced into multivariate models with a final model including all groups.

1. Patient characteristics: age, gender, body mass index, bilateral versus unilateral disease and disease duration.

2. Pain mechanisms: peripheral pain mechanisms (ie, signs of inflammation, morning stiffness symptoms and radiographic findings), central pain mechanisms (ie, other joint OA, comorbidities and pain severity).

3. Intervention characteristics: clinical setting (ie, location of intervention), aspirate volume, frequency of injection, volume of injection, and intra-articular injection approach (ie, medial vs lateral approach and use of ultrasound-guided injection).

4. Trial characteristics: blinding (patients, assessors or physicians), dropout rates, role of funder/sponsor (ie, pharmaceutical company), randomisation ratio, trial duration, single centre/multicentre study, parallel/cross-over trial and use of 'intention to treat' analysis.

The trials that originate the IPD will also be coded and included as a level variable in all analyses. ESs and 95\% CI will be generated for each outcome measure. $\mathrm{P}<0.05$ will be considered statistically significant.
A sensitivity analysis will be conducted using pain scores (instead of change in pain scores) as a continuous dependent variable and repeating the approaches described above.

Statistical analyses will be performed using Stata SE V.14.

\section{EXPECTED RESEARCH CONTRIBUTION}

It is envisaged that the investigators will deliver data to be used in the design and execution of future clinical trials. It will allow for better understanding of the placebo response and subsequent implementation of clinical designs with lowered placebo responses.

\section{ETHICS AND DISSEMINATION}

This study does not include identifiable data. Ethical approval was obtained by the original investigators. Results of the IPD meta-analysis will be disseminated for publication in a peer-reviewed journal and by international conference presentations.

\section{Author affiliations}

${ }^{1}$ Department of Rheumatology, Royal North Shore Hospital, University of Sydney, Sydney, New South Wales, Australia

2Department of Rheumatology, Institute of Bone and Joint Research, University of Sydney, St Leonards, New South Wales, Australia

${ }^{3}$ Erasmus MC University Medical Center, Rotterdam, The Netherlands

${ }^{4}$ Department of General Practice, Erasmus University Medical Centre, Rotterdam,

The Netherlands

${ }^{5}$ Division of Academic Rheumatology, University of Nottingham, Nottingham, Nottingham, UK

Contributors Study design: SP-CY, MLF, SMAB-Z, MvM, WZ and DJH contributed to the study design. SP-CY and LAD will be conducting the systematic review, data extraction and analysis. SP-CY drafted the first version of the manuscript and all the authors were involved in the critical revision of the manuscript for important intellectual content. The study proposal has been peer-reviewed and approved by the OA Trial Bank Steering Committee.

Funding SP-CY holds a University of Sydney Postgraduate Research Scholarship (Part Time). MLF holds a National Health and Medical Research Council (NHMRC) Career Development Fellowship and is a Sydney Medical Foundation Fellow. DJH holds an NHMRC Practitioner Fellowship. SMAB-Z reports grants from European Union, The Netherlands Organisation for Health Research and Development, Dutch Arthritis Foundation. WZ is supported by a grant from Arthritis Research UK. The OA Trial Bank is supported by the Dutch Arthritis Society.

Competing interests DJH reports personal fees from consulting fees from Merck Serono, Flexion and Tissuegene, outside the submitted work. All other authors have nothing to disclose.

Patient consent for publication Not required.

Provenance and peer review Not commissioned; externally peer reviewed.

Open access This is an open access article distributed in accordance with the Creative Commons Attribution Non Commercial (CC BY-NC 4.0) license, which permits others to distribute, remix, adapt, build upon this work non-commercially, and license their derivative works on different terms, provided the original work is properly cited, appropriate credit is given, any changes made indicated, and the use is non-commercial. See: http://creativecommons.org/licenses/by-nc/4.0/.

\section{REFERENCES}

1. Hunter DJ, Bowden JL. Therapy: Are you managing osteoarthritis appropriately? Nat Rev Rheumatol 2017;13:703-4. 
2. Hunter DJ, Schofield D, Callander E. The individual and socioeconomic impact of osteoarthritis. Nat Rev Rheumatol 2014;10:437-41.

3. Avouac J, Gossec L, Dougados M. Efficacy and safety of opioids for osteoarthritis: a meta-analysis of randomized controlled trials. Osteoarthritis Cartilage 2007;15:957-65.

4. Mitchell HL, Hurley MV. Management of chronic knee pain: a survey of patient preferences and treatment received. BMC Musculoskelet Disord 2008;9:123.

5. Gore M, Sadosky AB, Leslie DL, et al. Therapy switching, augmentation, and discontinuation in patients with osteoarthritis and chronic low back pain. Pain Pract 2012;12:457-68.

6. Machado GC, Maher CG, Ferreira PH, et al. Efficacy and safety of paracetamol for spinal pain and osteoarthritis: systematic review and meta-analysis of randomised placebo controlled trials. BMJ 2015;350:h1225.

7. Bennell KL, Hunter DJ, Hinman RS. Management of osteoarthritis of the knee. BMJ 2012;345:e4934.

8. McAlindon TE, Bannuru RR, Sullivan MC, et al. OARSI guidelines for the non-surgical management of knee osteoarthritis. Osteoarthritis Cartilage 2014;22:363-88.

9. Hochberg MC, Altman RD, April KT, et al. American College of Rheumatology 2012 recommendations for the use of nonpharmacologic and pharmacologic therapies in osteoarthritis of the hand, hip, and knee. Arthritis Care Res 2012;64:465-74.

10. Jevsevar DS, Brown GA, Jones DL, et al. The american academy of orthopaedic surgeons evidence-based guideline on: Treatment of osteoarthritis of the knee, 2nd edition. J Bone Joint Surg Am 2013:95:1885-6.

11. Finniss DG, Kaptchuk TJ, Miller F, et al. Biological, clinical, and ethical advances of placebo effects. Lancet 2010;375:686-95.

12. Saltzman BM, Leroux T, Meyer MA, et al. The therapeutic effect of intra-articular normal saline injections for knee osteoarthritis: A meta-analysis of evidence level 1 studies. Am J Sports Med 2017; 45:2647-53.

13. Altman RD, Devji T, Bhandari M, et al. Clinical benefit of intraarticular saline as a comparator in clinical trials of knee osteoarthritis treatments: A systematic review and meta-analysis of randomized trials. Semin Arthritis Rheum 2016;46:151-9.

14. Zhang W, Robertson J, Jones AC, et al. The placebo effect and its determinants in osteoarthritis: meta-analysis of randomised controlled trials. Ann Rheum Dis 2008;67:1716-23.

15. Zou K, Wong J, Abdullah N, et al. Examination of overall treatment effect and the proportion attributable to contextual effect in osteoarthritis: meta-analysis of randomised controlled trials. Ann Rheum Dis 2016;75:1964-70.

16. Abhishek A, Doherty M. Mechanisms of the placebo response in pain in osteoarthritis. Osteoarthritis Cartilage 2013;21:1229-35.

17. Riley RD, Lambert PC, Abo-Zaid G. Meta-analysis of individual participant data: rationale, conduct, and reporting. $B M J$ 2010;340:c221.

18. van Middelkoop M, Arden NK, Atchia I, et al. The OA Trial Bank: meta-analysis of individual patient data from knee and hip osteoarthritis trials show that patients with severe pain exhibit greater benefit from intra-articular glucocorticoids. Osteoarthritis Cartilage 2016:24:1143-52.
19. Fu Y, Persson MS, Bhattacharya A, et al. Identifying placebo responders and predictors of response in osteoarthritis: a protocol for individual patient data meta-analysis. Syst Rev 2016;5:183.

20. Shamseer L, Moher D, Clarke M, et al. Preferred reporting items for systematic review and meta-analysis protocols (PRISMA-P) 2015: elaboration and explanation. BMJ 2015;350:g7647.

21. Altman R, Asch E, Bloch D, et al. Development of criteria for the classification and reporting of osteoarthritis. Classification of osteoarthritis of the knee. Diagnostic and Therapeutic Criteria Committee of the American Rheumatism Association. Arthritis Rheum 1986;29:1039-49.

22. Zhang W, Doherty M, Peat G, et al. EULAR evidence-based recommendations for the diagnosis of knee osteoarthritis. Ann Rheum Dis 2010;69:483-9.

23. van Middelkoop M, Dziedzic KS, Doherty M, et al. Individual patient data meta-analysis of trials investigating the effectiveness of intra-articular glucocorticoid injections in patients with knee or hip osteoarthritis: an OA Trial Bank protocol for a systematic review. Syst Rev 2013;2:54

24. Moher D, Liberati A, Tetzlaff J, et al. Preferred reporting items for systematic reviews and meta-analyses: the PRISMA statement. BMJ 2009;339:b2535.

25. Runhaar J, Rozendaal RM, van Middelkoop M, et al. Subgroup analyses of the effectiveness of oral glucosamine for knee and hip osteoarthritis: a systematic review and individual patient data meta-analysis from the OA trial bank. Ann Rheum Dis 2017;76:1862-9.

26. Higgins JP, Altman DG, Gøtzsche PC, et al. The Cochrane Collaboration's tool for assessing risk of bias in randomised trials. BMJ 2011;343:d5928.

27. Higgins JPT, Green S. Cochrane Handbook for Systematic Reviews of Interventions: The Cochrane Collaboration, 5.1.0 (updated March 2011). 2011. http://handbook.cochrane.org.

28. Sterne JA, Gavaghan D, Egger M. Publication and related bias in meta-analysis: power of statistical tests and prevalence in the literature. J Clin Epidemiol 2000;53:1119-29.

29. Donders AR, van der Heijden GJ, Stijnen T, et al. Review: a gentle introduction to imputation of missing values. J Clin Epidemiol 2006;59:1087-91.

30. Koopman L, van der Heijden GJ, Hoes AW, et al. Empirical comparison of subgroup effects in conventional and individual patient data meta-analyses. Int J Technol Assess Health Care 2008;24:358-61.

31. Groenwold RH, Donders AR, van der Heijden GJ, et al. Confounding of subgroup analyses in randomized data. Arch Intern Med 2009;169:1532-4.

32. Tubach F, Ravaud P, Martin-Mola E, et al. Minimum clinically important improvement and patient acceptable symptom state in pain and function in rheumatoid arthritis, ankylosing spondylitis, chronic back pain, hand osteoarthritis, and hip and knee osteoarthritis: Results from a prospective multinational study. Arthritis Care Res 2012;64:1699-707.

33. Wandel S, Jüni $\mathrm{P}$, Tendal $\mathrm{B}$, et al. Effects of glucosamine, chondroitin, or placebo in patients with osteoarthritis of hip or knee: network meta-analysis. BMJ 2010;341:c4675. 